Supporting information

\title{
Surface oxidation of polymer 3D porous structures using chlorine dioxide radical gas
}

\author{
Kohei Kikkawa, Yu-I Hsu*, Xinnan Cui, Shunsuke Mizuno, Taka-Aki Asoh, Hiroshi \\ Uyama* \\ Department of Applied Chemistry, Graduate school of Engineering, Osaka University, \\ 2-1, Yamadaoka, Suita, Osaka 565-0871, Japan
}

\section{* Corresponding author}

Phone: +81-6-6879-7364

Fax: $+81-6-6879-7367$

YH: yuihsu@chem.eng.osaka-u.ac.jp

HU: uyama@chem.eng.osaka-u.ac.jp 
Table S1. Thermal properties of PMP pellet and PMP monolith (PMP-60-70).

\begin{tabular}{|l|l|l|l|l|}
\hline & $\Delta \mathrm{Hm}(\mathrm{J} / \mathrm{g})$ & $\mathrm{Tm}\left({ }^{\circ} \mathrm{C}\right)$ & $\Delta \mathrm{Hc}(\mathrm{J} / \mathrm{g})$ & $\mathrm{Tc}\left({ }^{\circ} \mathrm{C}\right)$ \\
\hline Pellet & 30.1 & 234.6 & -32.0 & 191 \\
\hline Monolith & 36.3 & 236.7 & -34.5 & 190 \\
\hline
\end{tabular}

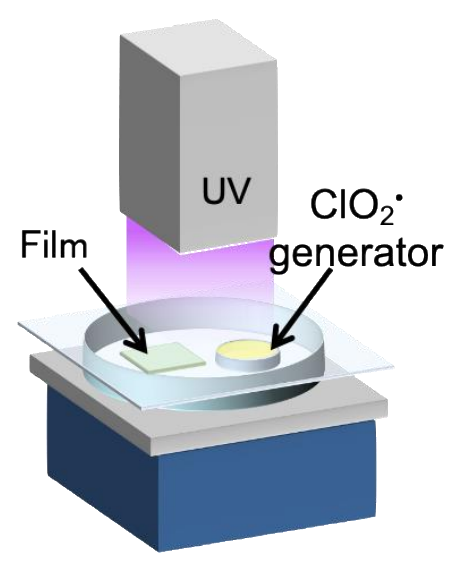

Figure S1. The illustration of the oxidation equipment of PMP film. 


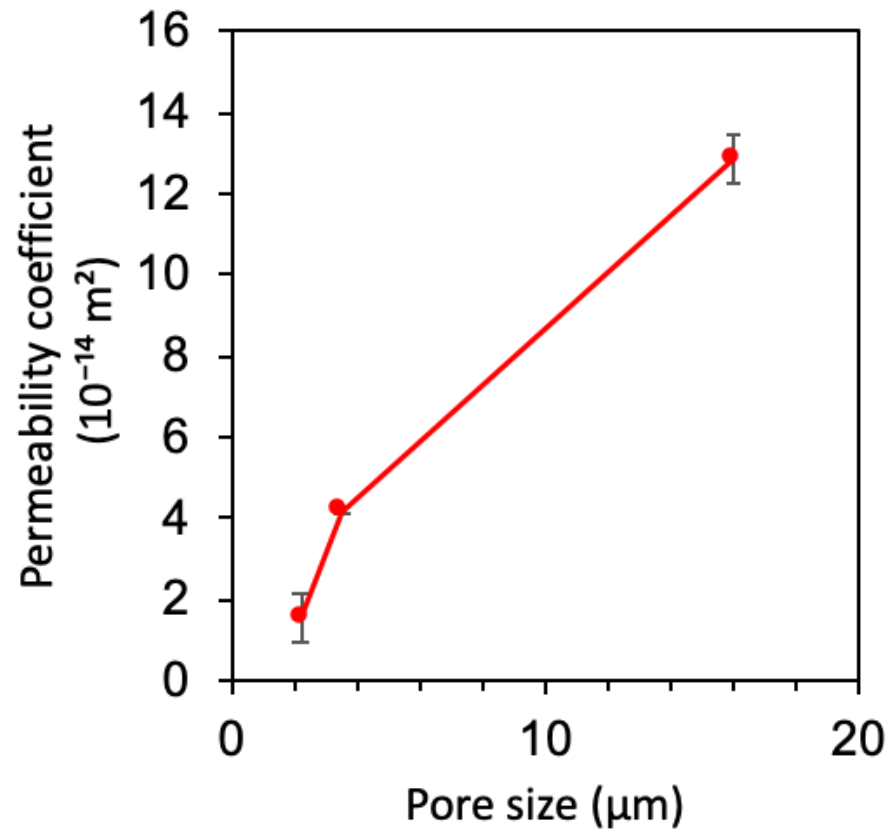

Figure S2. Relationship between water permeability and pore size.

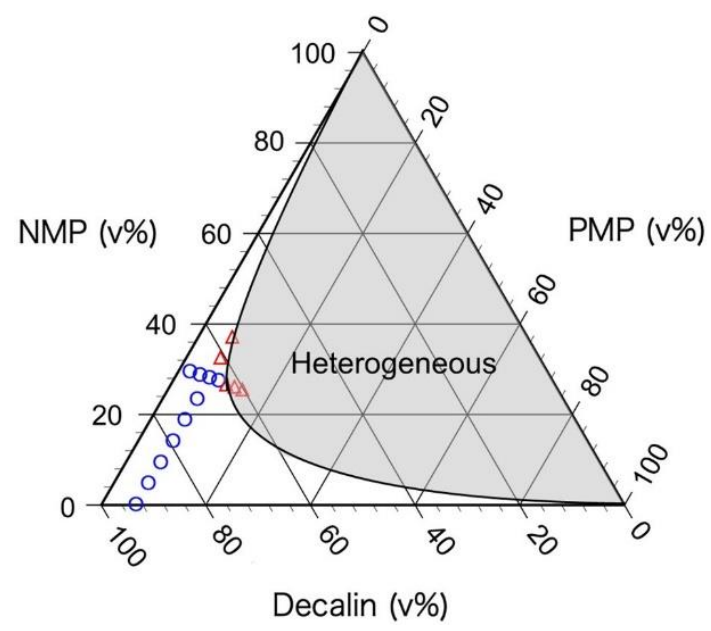

Figure S3. Ternary phase diagram of PMP/Decalin/NMP system at $120^{\circ} \mathrm{C}$. (Blue circle:

homogeneous state at $120^{\circ} \mathrm{C}$; Red triangle: heterogeneous state at $120^{\circ} \mathrm{C}$ ). 

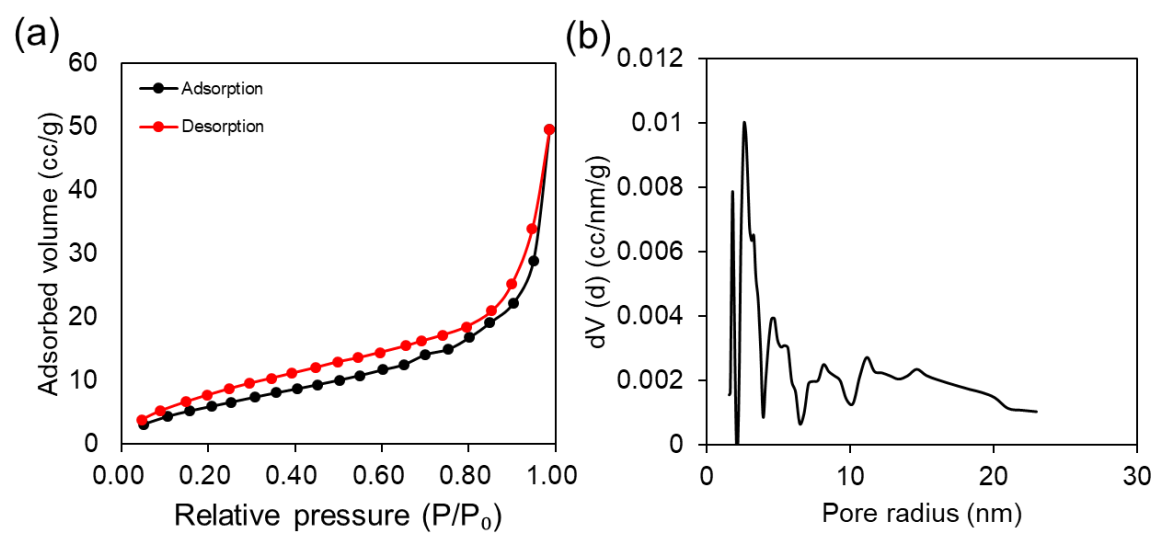

Figure S4. (a) $\mathrm{N}_{2}$ adsorption-desorption isotherm at $-196^{\circ} \mathrm{C}$ for PMP-60-70. (b) Pore size distribution of PMP-60-70. The pore size was analyzed using the desorption data and applying NLDFT method.

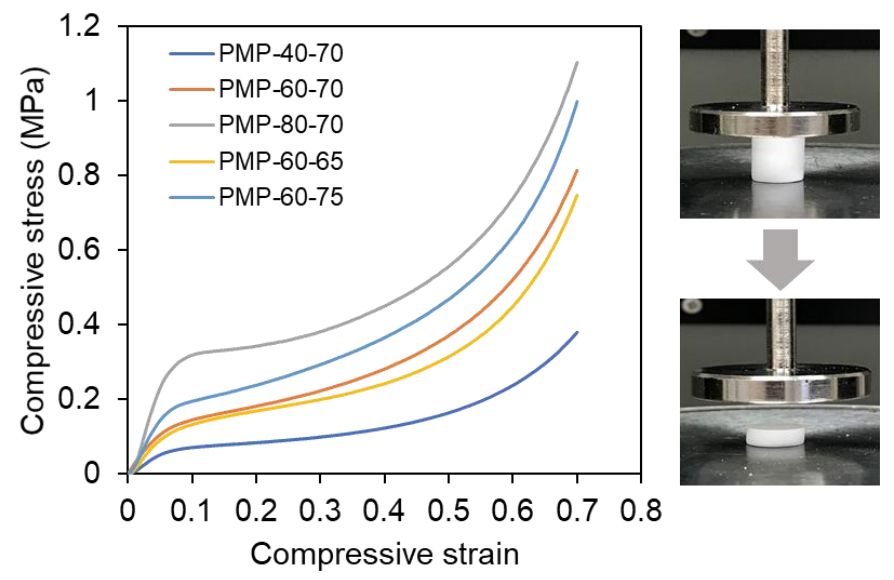

Figure S5. Compressive stress-strain curves of PMP monolith at a maximum strain of $70 \%$ of initial length. 


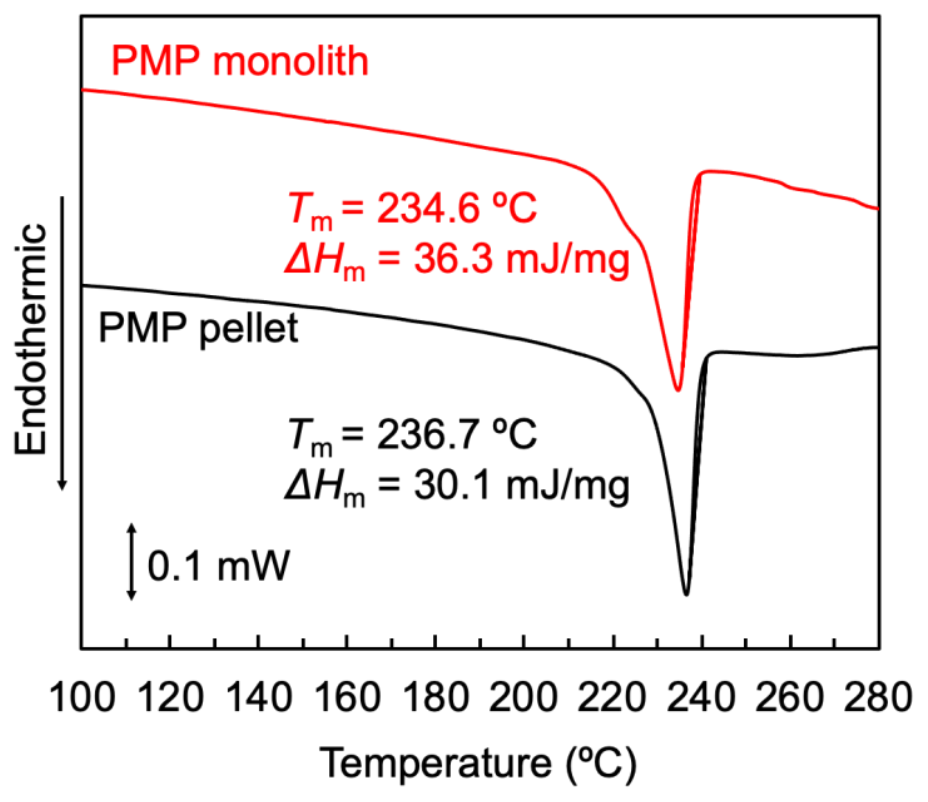

Figure S6. DSC thermograms of PMP pellet and monolith (PMP-60-70). 

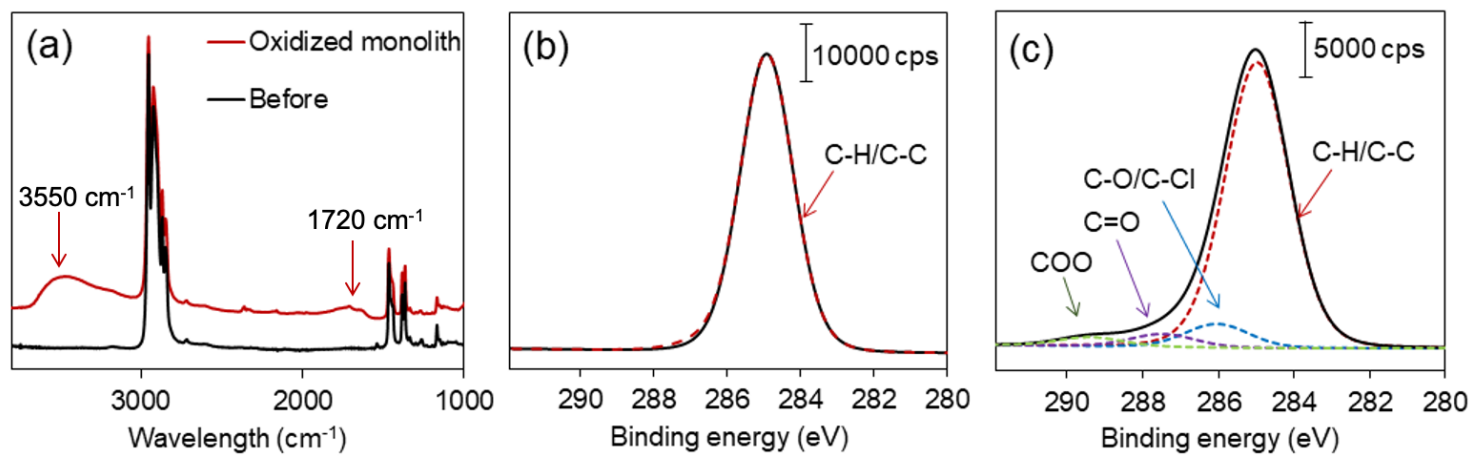

Figure S7. (a) FT-IR spectra of neat PMP (black) and oxidized PMP monolith (red).

XPS C1s spectra of PMP monolith (b) before and (c) after light-activated $\mathrm{ClO}_{2}{ }^{\circ}$ oxidation.

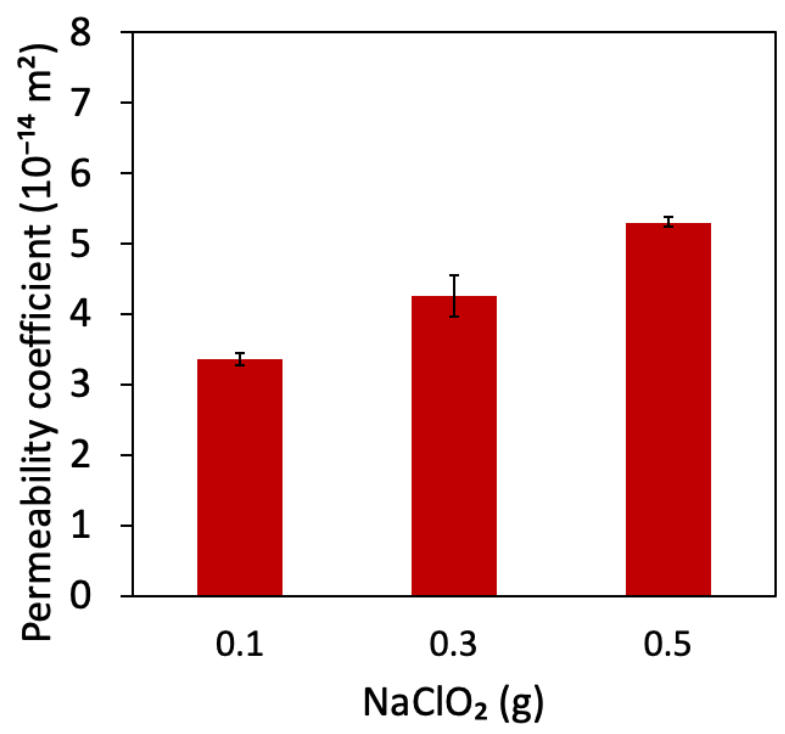

Figure S8. Relationship between water permeability coefficient and initial $\mathrm{NaClO}_{2}$ amount. 